\title{
Reversible Photoreduction as a Trigger for Photoresponsive Gels
}

\author{
Emily R. Draper, ${ }^{\dagger}$ Ralf Schweins, ${ }^{\ddagger}$ Riaz Akhtar, ${ }^{\S}$ Philip Groves, "Victor Chechik," \\ Martijn A. Zwijnenburg, ${ }^{\perp}$ and Dave J. Adams ${ }^{*}, \dagger$ \\ ${ }^{\dagger}$ Department of Chemistry, University of Liverpool, Crown Street, Liverpool L69 7ZD, United Kingdom \\ ${ }^{\ddagger}$ Institut Laue-Langevin, Large Scale Structures Group, 71 Avenue des Martyrs, CS 20156, F-38042 Grenoble CEDEX 9, France \\ ${ }^{\S}$ Centre for Materials and Structures, School of Engineering, University of Liverpool, Liverpool L69 3GH, United Kingdom \\ "Department of Chemistry, University of York, Heslington, York YO10 5DD, United Kingdom \\ ${ }^{\perp}$ Department of Chemistry, University College London, 20 Gordon Street, London WC1H 0AJ, United Kingdom
}

\section{Supporting Information}

\begin{abstract}
We present here a new type of photoresponsive, reversible low molecular weight gel. All previous examples rely on a photoisomerisation, ring-closing or dimerization. We show that photoreduction of a perylene bisimide gelator results in the formation of a stable radical anion. The formation of the radical anion results in a change in the packing of the perylene bisimides in the self-assembled aggregates, leading to a change in fibrous network and an increase in the rheological properties of the gels. An increase in the rheological properties is extremely rare for a photoresponsive gel; normally, irradiation results in a gel-to-sol transition, and the gel falling apart. As the radical anion decays, which takes several hours in air, the original gel properties are restored. This photoreduction can be cycled many times. Finally, we show that the mechanical properties are different between irradiated and nonirradiated sections in a patterned gel.
\end{abstract}

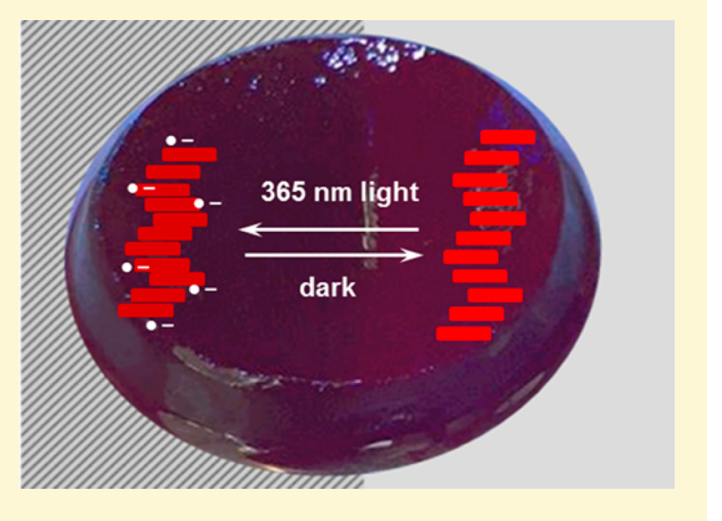

\section{INTRODUCTION}

Using light as a trigger for changing the properties of materials allows spatial resolution and hence controlled patterning. There are many light-responsive supramolecular structures that exploit the rapid response of such materials to irradiation. Numerous examples exist where specific chromophores that respond to irradiation are built into the supramolecular structures. One example is light-responsive low molecular weight gels. ${ }^{1-3}$ The vast majority of these are based around azobenzene. The transisomer is generally an effective low molecular weight gelator, while the cis-isomer is not. ${ }^{4,5}$ Irradiation of a gel results in a trans-to-cis isomerization, a gel-to-sol transition, and so the gel falls apart. Heating to recover the trans-isomer, followed by cooling usually results in the gel reforming. Hence, cycling the gel often requires an additional thermal step rather than just further irradiation. Analogous examples with stilbenes exist. ${ }^{6}$ Alternative light-responsive gelators are based on selfdimerization, which results in a change in the mechanical properties of the gel. Again, in most cases, dimerization leads to destruction of the gel, ${ }^{7,8}$ although this can also be used to stabilize the network. ${ }^{9}$ Finally, a photoinduced ring opening or closing can be used to change the gel's properties. For example, the photoinduced ring closing and opening of dithienylethenes can be used to prepare photochromic gels. ${ }^{10}$ Ring closing of spiropyrans has also been used. ${ }^{11}$

For all of these examples where irradiation leads to change in the rheological properties, the end result is generally the destruction of the gel. There are very few examples where irradiation leads to a strengthening of the gel network. Van Herpt et al. showed that a gel could be maintained as the color was changed via photoinduced ring-closure for a dithienylethene-based gel, although the rheological properties were not measured after irradiation. ${ }^{10}$ We recently demonstrated a rare exception where the mechanical properties of the gel were improved on irradiation, using the dimerization of a coumarinbased gelator. However, in this case, the change in properties was irreversible. ${ }^{9}$

Perylene bisimides (PBIs) can be used to form effective low molecular weight gelators. ${ }^{12,13}$ Amino acid functionalized PBIs can form hydrogels when the $\mathrm{pH}$ of a solution is decreased below the $\mathrm{p} K_{\mathrm{a}}$ of the terminal carboxylic acid. ${ }^{14,15}$ Above the $\mathrm{p} K_{a}$, worm-like micelles are typically formed. Reduction of PBIs to the radical anion or dianion is straightforward, ${ }^{16}$ either chemically by the addition of sodium dithionite or by photoirradiation. In solution, reaction of the reduced species with oxygen is trivial, and the radical anions have been used as oxygen sensors. ${ }^{17}$ However, in dried films of self-assembled PBIs, the radical anions are significantly more stable to oxygen; these species can persist for several hours in air. ${ }^{14}$

Received: July 1, 2016

Revised: August 19, 2016

Published: August 23, 2016 
Although isomerization, dimerization and ring-closing have all been used to affect and control the properties of low molecular weight gels, as far as we are aware there are no literature examples where photoreduction has been used as a method of increasing the mechanical properties of a gel. Chemical reduction has previously been used to change the mechanical properties of gels, but this caused the gel to fall apart. ${ }^{18}$ Chemical oxidation of a dihydropyridine precursor to a pyridine gelator has been shown to result in gel formation; the oxidation leads to planarization and donor-acceptor interactions. $^{19}$

\section{EXPERIMENTAL SECTION}

PBI 1 was synthesized as described previously. ${ }^{14}$ Doubly distilled deionized water was used throughout. All other chemicals were purchased from Sigma-Aldrich and used as received.

Gel Formation. $10 \mathrm{mg}$ of gelator 1 was dissolved using 1 mol equiv of $0.1 \mathrm{M}$ sodium hydroxide. Water was then added to give $2 \mathrm{~mL}$ of solution and a concentration of $5 \mathrm{mg} / \mathrm{mL}$ of gelator in solution. The solution was stirred to dissolve the gelator. This solution was then transferred to a vial containing a $6 \mathrm{mg}$ of glucono- $\delta$-lactone (GdL) and shaken gently. $1 \mathrm{~mL}$ was then transferred to a $20 \mathrm{~mL}$ plastic syringe with the top removed. The open syringe was covered with Parafilm and the solution was left to gel overnight. The gel was removed from the syringe using the plunger.

Irradiation. Gels were placed onto a glass microscope slide and placed inside a plastic Petri dish with a wet paper towel in to keep the air saturated with water. The lid of the Petri dish had a hole cut out to allow the LED to be able to irradiate the sample. A heat filter was then placed over this hole. A $365 \mathrm{~nm}$ LED or $490 \mathrm{~nm}$ LED (LedEngin Inc., LZ1-10U600) with a light source powered by a TTi QL564P power supply operating at $1.0 \mathrm{~W}$ was used. When using a mask, a shape was cut out of a sheet of opaque plastic and placed over the sample prior to irradiation.

Rheology. Dynamic rheological measurements were performed using an Anton Paar Physica MCR101 rheometer. A parallel measuring system was used. The gels were prepared as above and transferred onto the bottom plate. All experiments were performed at $25^{\circ} \mathrm{C}$. Strain sweeps were performed with a $25 \mathrm{~mm}$ plate from $0.1 \%$ to $100 \%$ with a frequency of $10 \mathrm{rad} / \mathrm{s}$, with a gap of $1.3 \mathrm{~mm}$. The critical strain was quoted as the point that $G^{\prime}$ starts to deviate for linearity.

UV-vis. Absorption Measurements were carried out on a Shimadzu UV-2550 UV-Vis spectrophotometer. Samples for UV-vis measurements were placed in a demountable $0.1 \mathrm{~mm}$ quartz cuvette and irradiated with a LED for $5 \mathrm{~min}$ and the spectra collected. Kinetic measurements were collected for $16 \mathrm{~h}$ measuring the absorbance at $720 \mathrm{~nm}$ every $15 \mathrm{~min}$.

SEM. SEM images were obtained using a Hitachi S-4800 FE-SEM at $3 \mathrm{keV}$. Gel was deposited onto glass coverslips which were fixed onto aluminum SEM stubs with carbon tabs and left to dry for $24 \mathrm{~h}$. The samples were gold coated for $3 \mathrm{~min}$ at $15 \mathrm{~mA}$ prior to imaging using a sputter coater (EMITECH K550X).

Small Angle Neutron Scattering. Solutions were prepared as above, but with the $\mathrm{H}_{2} \mathrm{O}$ and $\mathrm{NaOH}$ replaced with $\mathrm{D}_{2} \mathrm{O}$ and $\mathrm{NaOD}$ respectively. The gels were prepared in UV spectrophotometer grade, quartz cuvettes (Hellma) with a $2 \mathrm{~mm}$ path length, which were housed in a temperature controlled sample rack during the measurements. SANS measurements were performed using the D11 instrument (Institut Laue Langevin, Grenoble, France). A neutron beam, with a fixed wavelength of $10 \AA$ and divergence of $\Delta \lambda / \lambda=9 \%$, allowed measurements over a large range in $Q[Q=4 \pi \sin (\theta / 2) / \lambda]$ range of 0.01 to $0.3 \AA^{-1}$, by using two sample-detector distances.

The data were reduced to $1 \mathrm{D}$ scattering curves of intensity vs $Q$ using the facility provided software. The electronic background was subtracted, the full detector images for all data were normalized and scattering from the empty cell was subtracted. The scattering from $\mathrm{D}_{2} \mathrm{O}$ was also measured and subtracted from the data. The data were radially averaged to produce the $1 \mathrm{D}$ curves for each detector position.
The instrument-independent data were then fitted to the CorrLength model in the SasView software package. ${ }^{20}$

Density Functional Theory Calculations. Calculations were performed using the B3LYP+D3 hybrid density functional $2^{21-23}$ with empirical dispersion correction, the DZP ${ }^{24}$ and def2-TZVP ${ }^{25}$ basis-sets and the Turbomole 6.6 code. Calculations that modeled the fibers in an aqueous environment additionally employed the $\operatorname{COSMO}^{26}$ continuum solvation model with a relative dielectric permittivity $\left(\varepsilon_{\mathrm{r}}\right)$ of 80 .

Dynamic Nanoindentation. Dynamic nanoindentation was performed using a Keysight Technologies Nanoindenter G200 with a DCM-II Head. Measurements were conducted with a $100 \mu \mathrm{m}$ flat punch indenter (Synton-MDP Ltd., Nidau, Switzerland) at room temperature $\left(25{ }^{\circ} \mathrm{C}\right)$. The sample was placed in a custom sample holder (20 mm diameter, with a $5 \mathrm{~mm}$ well) and 10 indentations were made on the sample ( $5 \times 2$ array) with $400 \mu \mathrm{m}$ spacing between each indent. The refined surface detection methodology that we employed in our previous studies was used for testing. ${ }^{27}$ Immediately after completion of the indentations, the gel was kept in the sample puck and the side of the gel that had been indented was masked. It was then irradiated for $30 \mathrm{~min}$. The irradiated side was indented using the same approach.

\section{RESULTS AND DISCUSSION}

We have previously reported that $\mathbf{1}$ (Figure 1a) forms wormlike micelles at a concentration of $5 \mathrm{mg} / \mathrm{mL}$ above $\mathrm{pH} 7$ (above
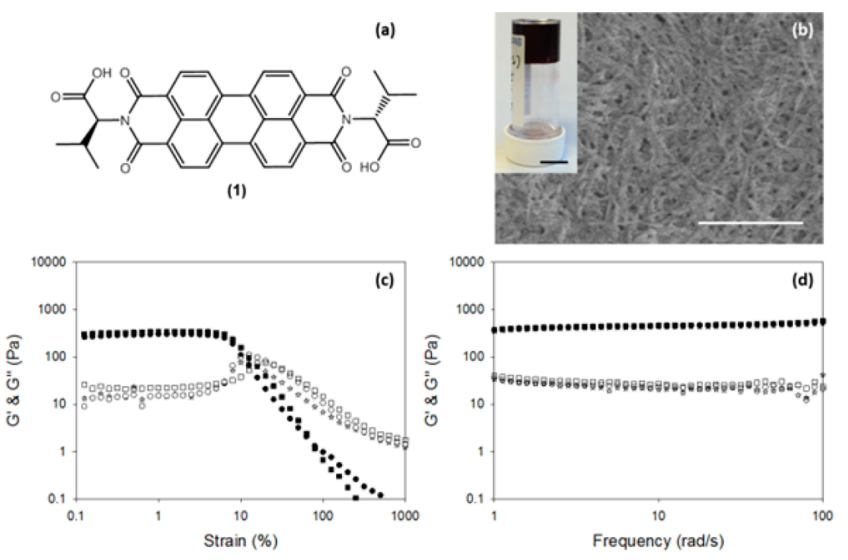

(OH

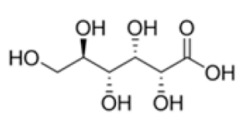

(e)

Figure 1. (a) Structure of gelator-1; (b) SEM of the fibrous structures formed in a gel using $\mathbf{1}$ at a concentration of $5 \mathrm{mg} / \mathrm{mL}$ and at $\mathrm{pH} 3.4$. Scale bar represents $500 \mathrm{~nm}$ (inset shows photograph of the gel. Scale bar represents $0.5 \mathrm{~cm}$ ); (c) Repeat rheological strain sweeps performed on gels of 1 performed at $10 \mathrm{rad} / \mathrm{s}$ at $25{ }^{\circ} \mathrm{C}$; (d) Frequency sweeps performed at $0.5 \%$ strain and $25{ }^{\circ} \mathrm{C}$. Different symbols represent data for different gels of 1 tested. Full symbols are $G^{\prime}$ and empty symbols are $G^{\prime \prime}$. (e) Scheme of the hydrolysis of glucono- $\delta$-lactone in water to gluconic acid.

the $\mathrm{p} K_{\mathrm{a}}$ of the carboxylic acids). ${ }^{14}$ On lowering the $\mathrm{pH}$ to 4 (i.e., below the $\mathrm{p} K_{\mathrm{a}}$ ) with glucono- $\delta$-lactone (GdL, Figure le), ${ }^{28,29}$ reproducible self-supporting hydrogels are formed as $\mathbf{1}$ self-assembles further into an entangled network (Figure 1b). The gels are typical low molecular weight gels; rheologically, they break at relatively low strain (3\%) and are essentially frequency independent with $G^{\prime}=480 \mathrm{~Pa}$ and $G^{\prime \prime}=25 \mathrm{~Pa}$ (Figure 1c,d).

Our previous work showed that the radical anion is formed when dried solutions or xerogels of $\mathbf{1}$ are irradiated with a 365 
nm LED. ${ }^{14}$ The choice of wavelength is important. Wavelengths larger than this do not result in formation of the radical anion despite the strong absorption of the PBI at $450 \mathrm{~nm}$. We explained this wavelength dependence in terms of needing to provide light of an energy greater than the optical gap + the exciton binding energy to form free charge carriers rather than excitons (bound electron-hole pairs). ${ }^{14}$

Irradiating the solution of worm-like micelles at $\mathrm{pH} 9$ in a sealed cuvette with a $365 \mathrm{~nm}$ LED results in the formation of the radical anion. This can be shown by UV-vis absorption spectroscopy, with the appearance of peaks at 720 and $810 \mathrm{~nm}$ (Figure S1a, Supporting Information) characteristic of the radical anion, ${ }^{16}$ and visually by a color change from red to a dark purple. The solution returns to a red color with time (Figure S1b-f, Supporting Information). Previously, (electro)chemical reduction has been used to form this radical anion; $^{30,31}$ to the best of our knowledge, this is the first demonstration of the in situ formation in solution using photoreduction. In a sealed cuvette, the radical anion persists for a significant amount of time. However, when the solution is exposed to air the radical anion decays quickly.

When the gel is irradiated in air, there is also a visible color change, which persists for hours (Figure $2 a-c$ ). There is also a
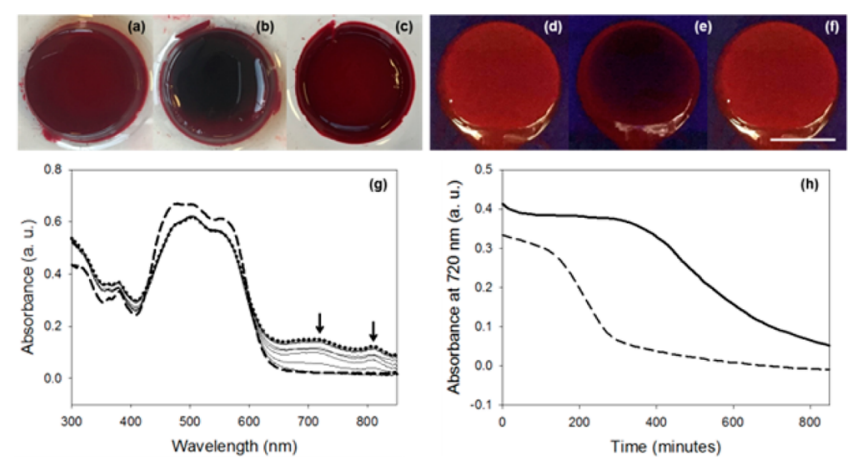

Figure 2. (a) Photograph of a gel of $\mathbf{1}$ prepared at $5 \mathrm{mg} / \mathrm{mL}$ and a $\mathrm{pH}$ of 3.4 in a mold and then removed; (b) after irradiation with a $365 \mathrm{~nm}$ LED for $30 \mathrm{~min}$ and (c) after $24 \mathrm{~h}$ photographed under natural light; panels $d-f$ show the gels in panels $a-c$ photographed under a handheld $365 \mathrm{~nm}$ UV light. Scale bar represents $1 \mathrm{~cm}$; (g) UV-vis spectra showing gel of 1 after irradiation with $365 \mathrm{~nm}$ (dotted line) relaxing after $18 \mathrm{~h}$ to the dashed line. The arrows show the peaks at 720 and $810 \mathrm{~nm}$, which decrease over time; (h) plot showing the change in absorbance at $720 \mathrm{~nm}$ overtime, and so the lifetime of the radical anion in the gel (solid data) and in solution (dashed data) as measured in a closed $0.1 \mathrm{~mm}$ cuvette.

loss in fluorescence (Figure $2 \mathrm{~d}-\mathrm{f}$ ). In the UV-vis spectrum, peaks appear again at 720 and $810 \mathrm{~nm}$; again due to the formation of the radical anion. ${ }^{16,32}$ EPR measurements confirm the presence of the radical anions (Figure S2, Supporting Information). ${ }^{33}$ We stress again that this is in the gel state and that these experiments were carried out in air, without degassing the solution or gel beforehand. Hence, the sample is exposed to oxygen, and one might expect that the radical anion would be quenched rapidly. ${ }^{17,34}$ This is not the case. We hypothesize that this is due to the nature of the self-assembled fibers in the gel, whereby the PBI radicals are stabilized by stacking as has been previously described. ${ }^{32}$

We suggest that diffusion of oxygen into the self-assembled structures is slow. Similar arguments have been made for the higher than expected stability of the radical anions in dried PBI films. ${ }^{32}$ Likewise, for naphthalene diimides (NDIs), it has been suggested that the stacking radical anions are more stable than the monomeric species since the oxygen cannot easily penetrate the stacks. ${ }^{35}$ For our system, in sealed cuvettes, the radical anion persists for over $14 \mathrm{~h}$ in the gel, as determined by UV-vis spectroscopy. However, it is difficult to directly correlate these time scales with the gels formed in molds for rheological testing (see below) since the geometry and exposure to air is different. However, in the gels formed in molds, the color persists for over $24 \mathrm{~h}$ (Figure S3, Supporting Information).

The formation of the radical anion correlates with a change in the mechanical properties of the hydrogel. Gels were formed in a mold as above, and then irradiated for specific lengths of time. The gels were then transferred to a rheometer. Irradiation led to an increase in the storage $\left(G^{\prime}\right)$ and loss $\left(G^{\prime \prime}\right)$ moduli as compared to a nonirradiated gel (Figure 3a and Figure S4, Supporting Information). The mechanical properties increased with the irradiation time (Figure $3 \mathrm{~b}$ and Figure S5, Supporting Information), gradually reaching a plateau after around $2 \mathrm{~h}$. For these gels, the absolute moduli increase with irradiation, as does the breakage strain (the strain at which $G^{\prime}$ deviates from linearity). The increase in the mechanical properties correlates with the presence of the radical anion. Over time, the radical anion decays (as discussed above); the rheological properties also recover over time, with the moduli returning to almost identical values to before irradiation (Figure S6, Supporting Information). Interestingly, the breakage strain does not return to the original value, implying that the network has been affected to some degree by the irradiation process. The irradiation and changes in rheological properties can be cycled many times (Figure 3c).

A water filter was used to ensure that there was no heating of the gel during irradiation. Drying of the sample was minimized using a hydrated chamber; control experiments showed that

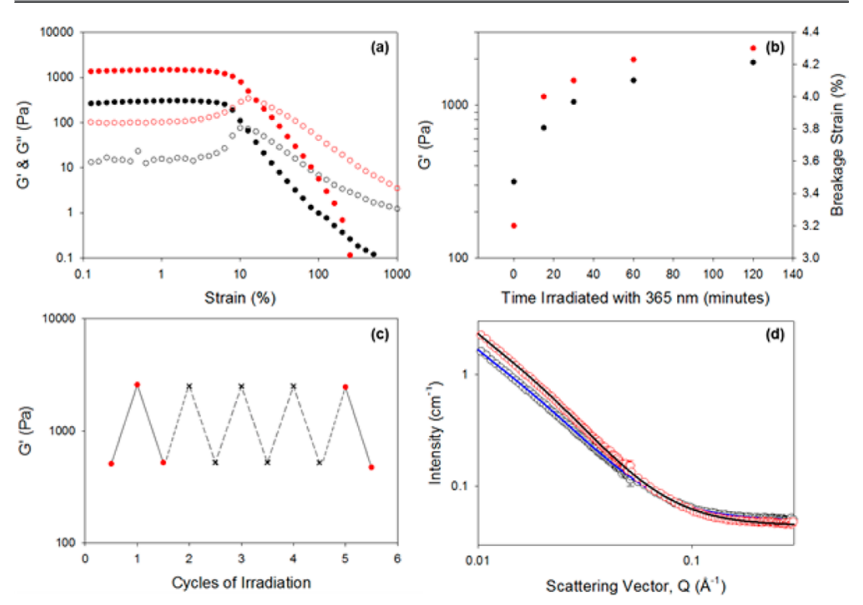

Figure 3. (a) Strain sweeps performed on gels of 1 (black data) compared to gels of 1 irradiated with a $365 \mathrm{~nm}$ LED for $1 \mathrm{~h}$ (red data) performed at $10 \mathrm{rad} / \mathrm{s}$ at $25{ }^{\circ} \mathrm{C}$; Full symbols are $G^{\prime}$ and empty symbols are $G^{\prime \prime}$; (b) Increase in $G^{\prime}$ plotted against irradiation time (data collected at $3 \%$ strain and $10 \mathrm{rad} / \mathrm{s}$, black data and left axis) and increase in breakage strain (where $G^{\prime}$ deviates from liberality) upon irradiation plotted against time (red data). Full symbols are $G^{\prime}$ and empty symbols are $G^{\prime \prime}$. (c) Rheology data showing $G^{\prime}$ at $10 \mathrm{rad} / \mathrm{s}$ after cycles of irradiation for $1 \mathrm{~h}$ and relaxation for $24 \mathrm{~h}$. Red data is recorded data from different samples. Crosses are hypothetical $G^{\prime}$ data. (d) SANS data collected before irradiation (black data) with fit (blue data) and after irradiation (red data) with fit (black data). 
sitting a sample in the chamber for set periods of time without irradiation did not lead to changes in the rheological data (Figure S7, Supporting Information). Irradiating with higher wavelength LEDs resulted in no change in the UV-vis spectrum (Figure S8a, Supporting Information), in line with our previous data where we showed that it was necessary to use a $365 \mathrm{~nm}$ LED to form the radical anion. Irradiating with higher wavelengths also did not lead to any change in the rheological data (Figure S8b, Supporting Information), again demonstrating that the presence of the radical anion results in the increase in gel stiffness.

A key question is how does the radical anion lead to an increase in the mechanical properties of the gel. Previous data has suggested conflicting ideas for the aggregation of radical anions. For a PBI-dimer based gelator, the formation of the radical anion chemically using sodium dithionite resulted in a gel-to-sol transition. ${ }^{18}$ This was attributed to an increase in water-solubility and electrostatic repulsion between PBIs. ${ }^{12,18}$ The gel reformed when the radical anion was quenched with oxygen. However, chemical reduction of a PBI in water had been shown to lead to further aggregation on the basis of the UV-vis spectra. ${ }^{16}$

To probe how the radical anion leads to changes in the rheological properties, the networks were probed in situ before and after irradiation using small angle neutron scattering (SANS). Recovery of the solutions was too fast to be amenable to this experiment. For the gels, there was a clear difference in the scattering before and after irradiation (Figure 3d). The data were fitted to the following functional form, based on related systems: ${ }^{36}$

$$
I(Q)=\frac{A}{Q^{n}}+\frac{C}{(1+(Q \xi))^{m}}+B
$$

The network scattering is described by the first term, whereas the second term describes the morphology of the fibrils and the nanoscale structure. Hence, $n$ and $m$ represent the fibril densities at low $q$ and high $q$ scattering, respectively. The correlation length, $\xi$, represents the distance between crosslinks. From the fits to the data, the low $q$ exponent $(n)$ does not change significantly before and after irradiation $(2.30 \pm 0.27$ and $2.29 \pm 0.07$, respectively). This implies that the network has a very similar morphology on these longer length scales. ${ }^{37}$ However, the high $q$ scattering exponent $(m)$ changes more significantly from $2.38 \pm 0.12$ before irradiation to $2.58 \pm 0.07$ after irradiation. There is also perhaps a slight decrease in the correlation length $(\xi)$ on irradiation from $6.25 \pm 0.61 \mathrm{~nm}$ to $5.91 \pm 0.23 \mathrm{~nm}$. Together, these data imply that the morphology on a larger length scale is not greatly affected by irradiation, but the entanglement density is affected; the distance between cross-links becoming smaller and the fiber density increases on the local scale. This correlates with the changes in the rheological data.

Further insight into the effect of irradiation on the fibers comes from computational work. DFT $+\mathrm{D}(\mathrm{B} 3 \mathrm{LYP}+\mathrm{D} 3)^{21-23}$ calculations, discussed in more detail in the Supporting Information, show that the most likely microscopic structure of the fibers involves bundles of stacks of PBI molecules with a spiral staircase motive (Figure $4 \mathrm{a}$ ), where every molecule is rotated, perpendicular to the stacking direction relative to the molecule below. The PBI tectons interact through $\pi-\pi$ stacking and by intermolecular hydrogen bonds involving the protons of the carboxylic acid groups of one molecule and the carbonyl oxygen atoms of the next molecule. A similar rotated structure

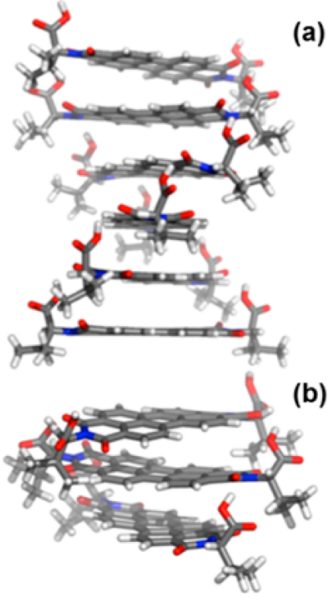

(a)

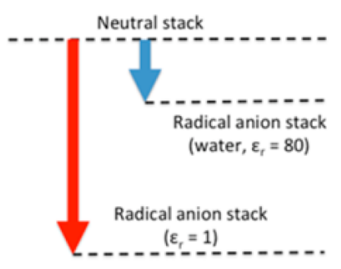

(c)

Figure 4. (a) Fragment of a stack of 1 optimized with the MMFF94 force field, ${ }^{41}$ clearly showing the spiral staircase stacking motive; (b) example of a trimer cluster model of a stack of $\mathbf{1}$ used in the DFT+D $(\mathrm{B} 3 \mathrm{LYP}+\mathrm{D} 3)^{21-23}$ calculations; (c) graphical illustration of the predicted change in intermolecular binding energy of a stack after localization of an excess electron, as calculated using B3LYP+D3 for a trimer cluster model of 1 in water (blue, $\varepsilon_{\mathrm{r}}=80$ ) and a low relative dielectric permittivity environment (red, $\varepsilon_{\mathrm{r}}=1$ ) respectively (see also Table S1, Supporting Information); (d) visualization of the B3LYP +D3 singly occupied molecular orbital of the radical anion trimer of 1 in an aqueous environment clearly showing the predicted localization of the excess electron in the middle of the stack.

was previously predicted ${ }^{38}$ by DFT $+\mathrm{D}$ calculations for a dimer of underivativised PBI, where no hydrogen bonds are present. Localizing an excess electron on cluster models of these stacks, i.e. forming the radical anion, results in a significant increase of the binding energy between the radical anion PBI and adjacent molecules. The exact degree of extra stabilization depends on the dielectric environment of the fiber $(>20$ and $>69 \mathrm{~kJ} / \mathrm{mol}$ for fibers in water and in low relative dielectric permittivity environments respectively, see Figure $4 \mathrm{c}$ and Table S1, Supporting Information). This increase in the intermolecular binding energy appears to be primarily driven by the attractive anion $-\pi$ interaction ${ }^{39}$ between the charge of the excess electron of the radical anion PBI molecule and the positive quadruple moments in the stacking direction of adjacent molecules. As long as the density of radical anions is low enough to minimize the number of close anion-anion pairs, this anion $-\pi$ interaction explains both the improved mechanical properties of the gels after illumination and that the original properties are recovered after the radical anions are quenched. Presumably, the changes in the entanglement density observed by SANS (above) are a result of these interactions leading to both changes in the inter- and intrafiber interactions. Exactly which interactions lead most to the improved mechanical properties is difficult to ascertain. The decreasing improvement in the mechanical properties with increasing illumination time (Figure S5, Supporting Information) then is the result of increasing numbers of destabilizing close anion-anion pairs, and/or the fact that the system is approaching equilibrium in terms of radical anion formation versus quenching (as suggested by the EPR data as a function of time in Figure S2b, Supporting Information). Alternatively, this could also be due to decrease in penetration of the light due to changes in the absorption spectrum. Finally, calculations show that the excess 
electron of the radical anion preferentially localizes in the middle of the stack (see Figure 4d).

This localization and the resulting reduced access to the unpaired electron by oxygen might also explain the relative stability of the radical anion of self-assembled PBI versus PBI in solution. We note that a positive charge (radical cation) must also be formed; we do not observe this radical cation, perhaps because it is UV signature is hidden by that of the radical anion. ${ }^{40}$ However, the radical cation and radical anion cannot reside too close together given the long lifetime of the radical anion and the effect of the radical cation on the strength of the stacks is predicted to be approximately neutral (Supporting Information).

Using light as a trigger allows spatial patterning. Using a mask, the radical anion is only formed in irradiated areas, correlating with a visible change in the color (Figure 5a). Using
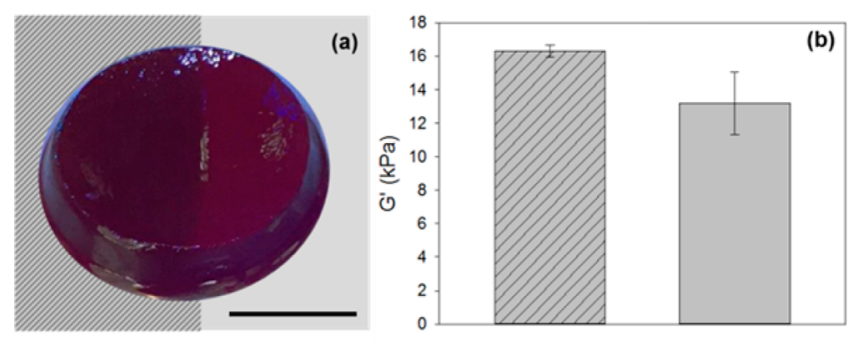

Figure 5. (a) Photograph of a gel which has only been irradiated in one area (the left hemisphere), as shown by the difference in color. The background has been added for clarity. (b) Dynamic nanoindentation data for the irradiated and nonirradiated sections of the gels. The error bars show the standard deviation over 10 and 8 individual measurements on the irradiated and nonirradiated regions on the gel, respectively.

dynamic nanoindentation, ${ }^{27}$ we probed locally the micromechanical behavior of the gels (Figure $5 b$ ) and found that the irradiated areas of the patterned gels have higher moduli than the nonirradiated areas. Additionally, the irradiated areas are more homogeneous (lower standard deviation) compared to the nonirradiated areas. Hence, this patterning approach could be used to prepare gels with spatially located sections with higher mechanical properties. The mechanical properties of gels such as this are important for both applications where specific mechanical strength is needed, and also for some functional purposes. For example, stem cell specification has been shown to be very sensitive to the elasticity of the hydrogel matrix. ${ }^{42,43}$ The rate of diffusion of encapsulated materials should also be affected by the gel matrix. ${ }^{44,45}$ Hence, one could imagine preparing gels where the mechanical properties were different in different regions, allowing specific routes through the bulk materials with different mechanical strengths or transport properties.

\section{CONCLUSIONS}

There are many photoresponsive gelators, but it is very rare for irradiation to lead to an increase in the mechanical properties of the gels. Usually, irradiation results in the gel falling apart, which is not always the desired response. Increasing the mechanical properties of the gel in a spatially controlled manner through patterning is therefore highly unusual. We show here that the formation of the radical anion for a PBI is possible using light; to the best of our knowledge, this is the first time that this has been demonstrated in the gel phase. This leads to an increase in the mechanical properties of the gels. The radical is surprisingly stable in the gel despite the presence of oxygen, lasting several hours and the improvement in gel properties persist over the same time scale. Our approach opens up spatially controlled increases in the mechanical properties of gels. We also note PBIs are widely used in optoelectronic applications and this work impacts on this, too.

\section{ASSOCIATED CONTENT}

\section{S Supporting Information}

The Supporting Information is available free of charge on the ACS Publications website at DOI: 10.1021/acs.chemmater.6b02677.

Additional experimental detail, UV-vis spectra, rheological data, and further computational information (PDF).

\section{AUTHOR INFORMATION}

\section{Corresponding Author}

*D. J. Adams. Email: d.j.adams@liverpool.ac.uk.

\section{Notes}

The authors declare no competing financial interest.

\section{ACKNOWLEDGMENTS}

E.D. thanks the EPSRC for funding (EP/L021978/1). M.Z. and D.A. thank the EPSRC for Fellowships (EP/I004424/1 and EP/L021978/1 respectively). The experiment at the Institut Laue Langevin was allocated beam time under experiment number 9-11-1756. This work benefitted from the SasView software, originally developed by the DANSE project under NSF award DMR-0520547. We thank Tom McDonald (University of Liverpool) for collecting the SEM data. We thank Alex Cowan (University of Liverpool) for helpful discussions.

\section{REFERENCES}

(1) Babu, S. S.; Praveen, V. K.; Ajayaghosh, A. Functional $\pi$-Gelators and Their Applications. Chem. Rev. 2014, 114, 1973-2129.

(2) Draper, E. R.; Adams, D. J. Responsive Materials by the Selfassembly of Low Molecular Weight Gelators. In Chemoresponsive Materials: Stimulation by Chemical and Biological Signals; Schneider, H.J., Ed.; Royal Society of Chemistry, 2015; Chapter 12, pp 332-363.

(3) Draper, E. R.; Adams, D. J. Photoresponsive gelators. Chem. Commun. 2016, 52, 8196-8206.

(4) Tamesue, S.; Takashima, Y.; Yamaguchi, H.; Shinkai, S.; Harada, A. Photoswitchable Supramolecular Hydrogels Formed by Cyclodextrins and Azobenzene Polymers. Angew. Chem., Int. Ed. 2010, 49, 7461-7464.

(5) Huang, Y.; Qiu, Z.; Xu, Y.; Shi, J.; Lin, H.; Zhang, Y. Supramolecular hydrogels based on short peptides linked with conformational switch. Org. Biomol. Chem. 2011, 9, 2149-2155.

(6) Eastoe, J.; Sanchez-Dominguez, M.; Wyatt, P.; Heenan, R. K. A photo-responsive organogel. Chem. Commun. 2004, 2608-2609.

(7) Kim, S. H.; Sun, Y.; Kaplan, J. A.; Grinstaff, M. W.; Parquette, J. $\mathrm{R}$. Photo-crosslinking of a self-assembled coumarin-dipeptide hydrogel. New J. Chem. 2015, 39, 3225-3228.

(8) Furman, I.; Weiss, R. G. Factors influencing the formation of thermally reversible gels comprised of cholesteryl 4-(2-anthryloxy)butanoate in hexadecane, 1-octanol, or their mixtures. Langmuir 1993, 9, 2084-2088.

(9) Draper, E. R.; McDonald, T. O.; Adams, D. J. Photodimerisation of a coumarin-dipeptide gelator. Chem. Commun. 2015, 51, 1282712830. 
(10) van Herpt, J. T.; Stuart, M. C. A.; Browne, W. R.; Feringa, B. L. A Dithienylethene-Based Rewritable Hydrogelator. Chem. - Eur. J. 2014, 20, 3077-3083.

(11) Qiu, Z.; Yu, H.; Li, J.; Wang, Y.; Zhang, Y. Spiropyran-linked dipeptide forms supramolecular hydrogel with dual responses to light and to ligand-receptor interaction. Chem. Commun. 2009, 3342-3344.

(12) Würthner, F.; Saha-Möller, C. R.; Fimmel, B.; Ogi, S.; Leowanawat, P.; Schmidt, D. Perylene Bisimide Dye Assemblies as Archetype Functional Supramolecular Materials. Chem. Rev. 2016, 116, 962-1052.

(13) Görl, D.; Zhang, X.; Würthner, F. Molecular Assemblies of Perylene Bisimide Dyes in Water. Angew. Chem., Int. Ed. 2012, 51, 6328-6348.

(14) Draper, E. R.; Walsh, J. J.; McDonald, T. O.; Zwijnenburg, M. A.; Cameron, P. J.; Cowan, A. J.; Adams, D. J. Air-stable photoconductive films formed from perylene bisimide gelators. $J$. Mater. Chem. C 2014, 2, 5570-5575.

(15) Roy, S.; Kumar Maiti, D.; Panigrahi, S.; Basak, D.; Banerjee, A. A new hydrogel from an amino acid-based perylene bisimide and its semiconducting, photo-switching behaviour. RSC Adv. 2012, 2, $11053-11060$

(16) Marcon, R. O.; Brochsztain, S. Aggregation of 3,4,9,10Perylenediimide Radical Anions and Dianions Generated by Reduction with Dithionite in Aqueous Solutions. J. Phys. Chem. A 2009, 113, 1747-1752.

(17) Shin, I.-S.; Hirsch, T.; Ehrl, B.; Jang, D.-H.; Wolfbeis, O. S.; Hong, J.-I. Efficient Fluorescence "Turn-On” Sensing of Dissolved Oxygen by Electrochemical Switching. Anal. Chem. 2012, 84, 91639168.

(18) Krieg, E.; Shirman, E.; Weissman, H.; Shimoni, E.; Wolf, S. G.; Pinkas, I.; Rybtchinski, B. Supramolecular Gel Based on a Perylene Diimide Dye: Multiple Stimuli Responsiveness, Robustness, and Photofunction. J. Am. Chem. Soc. 2009, 131, 14365-14373.

(19) Draper, E. R.; Eden, E. G. B.; McDonald, T. O.; Adams, D. J. Spatially resolved multicomponent gels. Nat. Chem. 2015, 7, 848-852.

(20) Grimme, S.; Antony, J.; Ehrlich, S.; Krieg, H. A consistent and accurate $\mathrm{ab}$ initio parametrization of density functional dispersion correction (DFT-D) for the 94 elements H-Pu. J. Chem. Phys. 2010, $132,154104$.

(21) Stephens, P. J.; Devlin, F. J.; Chabalowski, C. F.; Frisch, M. J. Ab Initio Calculation of Vibrational Absorption and Circular Dichroism Spectra Using Density Functional Force Fields. J. Phys. Chem. 1994, 98, 11623-11627.

(22) Becke, A. D. Density-functional thermochemistry. III. The role of exact exchange. J. Chem. Phys. 1993, 98, 5648-5652.

(23) Schäfer, A.; Horn, H.; Ahlrichs, R. Fully optimized contracted Gaussian basis sets for atoms Li to Kr. J. Chem. Phys. 1992, 97, 25712577.

(24) Weigend, F.; Ahlrichs, R. Balanced basis sets of split valence, triple zeta valence and quadruple zeta valence quality for $\mathrm{H}$ to $\mathrm{Rn}$ : Design and assessment of accuracy. Phys. Chem. Chem. Phys. 2005, 7, 3297-3305.

(25) Klamt, A.; Schuurmann, G. COSMO: a new approach to dielectric screening in solvents with explicit expressions for the screening energy and its gradient. J. Chem. Soc., Perkin Trans. 2 1993, 799-805.

(26) Draper, E. R.; Mears, L. L. E.; Castilla, A. M.; King, S. M.; McDonald, T. O.; Akhtar, R.; Adams, D. J. Using the hydrolysis of anhydrides to control gel properties and homogeneity in $\mathrm{pH}$-triggered gelation. RSC Adv. 2015, 5, 95369-95378.

(27) Adams, D. J.; Butler, M. F.; Frith, W. J.; Kirkland, M.; Mullen, L.; Sanderson, P. A new method for maintaining homogeneity during liquid-hydrogel transitions using low molecular weight hydrogelators. Soft Matter 2009, 5, 1856-1862.

(28) Pocker, Y.; Green, E. Hydrolysis of D-glucono-.delta.-lactone. I. General acid-base catalysis, solvent deuterium isotope effects, and transition state characterization. J. Am. Chem. Soc. 1973, 95, 113-119.
(29) Shirman, E.; Ustinov, A.; Ben-Shitrit, N.; Weissman, H.; Iron, M. A.; Cohen, R.; Rybtchinski, B. Stable Aromatic Dianion in Water. J. Phys. Chem. B 2008, 112, 8855-8858.

(30) Wilson, T. M.; Zeidan, T. A.; Hariharan, M.; Lewis, F. D.; Wasielewski, M. R. Electron Hopping among Cofacially Stacked Perylenediimides Assembled by Using DNA Hairpins. Angew. Chem., Int. Ed. 2010, 49, 2385-2388.

(31) Shirman, E.; Ustinov, A.; Ben-Shitrit, N.; Weissman, H.; Iron, M. A.; Cohen, R.; Rybtchinski, B. Stable Aromatic Dianion in Water. J. Phys. Chem. B 2008, 112, 8855.

(32) Seifert, S.; Schmidt, D.; Wurthner, F. An ambient stable coresubstituted perylene bisimide dianion: isolation and single crystal structure analysis. Chem. Sci. 2015, 6, 1663.

(33) Marcon, R. O.; Brochsztain, S. Highly Stable 3,4,9,10Perylenediimide Radical Anions Immobilized in Robust Zirconium Phosphonate Self-Assembled Films. Langmuir 2007, 23, 11972.

(34) Che, Y.; Yang, X.; Liu, G.; Yu, C.; Ji, H.; Zuo, J.; Zhao, J.; Zang, L. Ultrathin n-Type Organic Nanoribbons with High Photoconductivity and Application in Optoelectronic Vapor Sensing of Explosives. J. Am. Chem. Soc. 2010, 132, 5743.

(35) Miller, L. L.; Zhong, C. J.; Kasai, P. Production of a polymer with highly anisotropic conductivity and structure by Co-electroprecipitation of an imide anion radical and polycation. J. Am. Chem. Soc. 1993, 115, 5982.

(36) Hule, R. A.; Nagarkar, R. P.; Altunbas, A.; Ramay, H. R.; Branco, M. C.; Schneider, J. P.; Pochan, D. J. Correlations between structure, material properties and bioproperties in self-assembled [small beta]hairpin peptide hydrogels. Faraday Discuss. 2008, 139, 251.

(37) Ramachandran, S.; Trewhella, J.; Tseng, Y.; Yu, Y. B. Coassembling Peptide-Based Biomaterials: Effects of Pairing Equal and Unequal Chain Length Oligopeptides. Chem. Mater. 2006, 18, 6157.

(38) Zhao, H.-M.; Pfister, J.; Settels, V.; Renz, M.; Kaupp, M.; Dehm, V. C.; Würthner, F.; Fink, R. F.; Engels, B. Understanding Groundand Excited-State Properties of Perylene Tetracarboxylic Acid Bisimide Crystals by Means of Quantum Chemical Computations. J. Am. Chem. Soc. 2009, 131, 15660.

(39) Dawson, R. E.; Hennig, A.; Weimann, D. P.; Emery, D.; Ravikumar, V.; Montenegro, J.; Takeuchi, T.; Gabutti, S.; Mayor, M.; Mareda, J.; Schalley, C. A.; Matile, S. Experimental evidence for the functional relevance of anion $-\pi$ interactions. Nat. Chem. 2010, $2,533$.

(40) Ford, W. E.; Hiratsuka, H.; Kamat, P. V. Photochemistry of 3,4,9,10-perylenetetracarboxylic dianhydride dyes. 4. Spectroscopic and redox properties of oxidized and reduced forms of the bis(2,5-ditert-butylphenyl)imide derivative. J. Phys. Chem. 1989, 93, 6692.

(41) Halgren, T. A. Merck molecular force field. I. Basis, form, scope, parameterization, and performance of MMFF94. J. Comput. Chem. 1996, 17, 490.

(42) Engler, A. J.; Sen, S.; Sweeney, H. L.; Discher, D. E. Matrix Elasticity Directs Stem Cell Lineage Specification. Cell 2006, 126, 677.

(43) Alakpa, E. V.; Jayawarna, V.; Lampel, A.; Burgess, K. V.; West, C. C.; Bakker, S. C. J.; Roy, S.; Javid, N.; Fleming, S.; Lamprou, D. A.; Yang, J.; Miller, A.; Urquhart, A. J.; Frederix, P. W. J. M.; Hunt, N. T.; Péault, B.; Ulijn, R. V.; Dalby, M. J. Tunable Supramolecular Hydrogels for Selection of Lineage-Guiding Metabolites in Stem Cell Cultures. Chem. 2016, 1, 298-319.

(44) Wallace, M.; Adams, D. J.; Iggo, J. A. Analysis of the mesh size in a supramolecular hydrogel by PFG-NMR spectroscopy. Soft Matter 2013, 9, 5483.

(45) Branco, M. C.; Pochan, D. J.; Wagner, N. J.; Schneider, J. P. Macromolecular diffusion and release from self-assembled $\beta$-hairpin peptide hydrogels. Biomaterials 2009, 30, 1339. 